\section{Lenalidomide-based induction and maintenance in elderly newly diagnosed multiple myeloma patients: updated results of the EMNO1 ran- domized trial}

\author{
Sara Bringhen, ${ }_{1}^{1}$ Mattia D'Agostino, ${ }^{1}$ Laura Paris, ${ }^{2}$ Stelvio Ballanti, ${ }^{3}$ Norbert \\ Pescosta, ${ }^{4}$ Stefano Spada, ${ }^{1}$ Sara Pezzatti, ${ }^{5}$ Mariella Grasso, ${ }^{6}$ Delia Rota- \\ Scalabrini, ${ }^{7}$ Luca De Rosa, ${ }^{8}$ Vincenzo Pavone, ${ }^{9}$ Giulia Gazzera, ${ }^{1}$ Sara Aquino, ${ }^{10}$ \\ Marco Poggiu, ${ }^{1}$ Armando Santoro, ${ }^{11}$ Massimo Gentile, ${ }^{12}$ Luca Baldini, ${ }^{13}$ Maria \\ Teresa Petrucci, ${ }^{14}$ Patrizia Tosi, ${ }^{15}$ Roberto Marasca, ${ }^{16}$ Claudia Cellini, ${ }^{17}$ Antonio \\ Palumbo, ${ }^{10}$ Patrizia Falco, ${ }^{18}$ Roman Hájek, ${ }^{19,20}$ Mario Boccadoro ${ }^{1}$ and Alessandra \\ Larocca $^{1}$
}

\begin{abstract}
${ }^{1}$ Myeloma Unit, Division of Hematology, University of Torino, Azienda Ospedaliero-

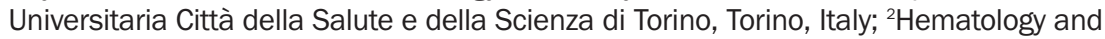
Bone Marrow Transplant Unit, Azienda Socio Sanitaria Territoriale Papa Giovanni XXIII, Bergamo, Italy; ${ }^{3}$ Sezione di Ematologia e Immunologia Clinica, Ospedale Santa Maria della Misericordia, località Sant'Andrea delle Fratte, Perugia, Italy; ${ }^{4}$ Reparto Ematologia e Centro TMO, Ospedale Centrale, Bolzano, Italy; ${ }^{5}$ Divisione di Ematologia, Ospedale S. Gerardo, Monza, Italy; ${ }^{6}$ Azienda Ospedaliera S. Croce-Carle, Cuneo, Italy; ${ }^{7}$ Medical Oncology, Candiolo Cancer Institute FPO-IRCCS, Candiolo, Italy; ${ }^{8} \mathrm{Hematology}$ and Stem Cell Transplantation Unit, Az. Osp. S. Camillo-Forlanini, Rome, Italy; ' UOC Ematologia e Trapianto, Az. Osp. C. Panico, Tricase (Lecce), Italy; ${ }^{10} \mathrm{Ematologia}$ e Centro Trapianti, IRCCS Ospedale Policlinico San Martino, Genova, Italy; ${ }^{11}$ Istituto Clinico Humanitas, Humanitas University, Rozzano-Milano, Italy; ${ }^{12} \mathrm{UOC}$ Ematologia AO Cosenza, Cosenza, Italy; ${ }^{13} \mathrm{UOC}$ Ematologia, Università degli Studi di Milano, Fondazione IRCCS Cà Granda, Ospedale Maggiore Policlinico, Milano, Italy; ${ }^{14} \mathrm{Hematology}$, Azienda Policlinico Umberto I, Roma, Italy; ${ }^{15}$ UO Ematologia, Ospedale di Rimini, AUSL della Romagna, Rimini, Italy; ${ }^{16} \mathrm{Hematology}$ Unit, Department of Medical and Surgical Sciences, University of Modena and Reggio Emilia, Modena, Italy; ${ }^{17}$ U.O. Ematologia, Ospedale Santa Maria delle Croci, Ravenna, Italy. ${ }^{18}$ SSD Ematologia, ASLTO4, Ospedali di Chivasso Cirié Ivrea, Italy; ${ }^{19}$ Department of Hematooncology, University Hospital Ostrava, Ostrava, Czech Republic and ${ }^{20}$ Faculty of Medicine, University of Ostrava, Ostrava, Czech Republic
\end{abstract}

${ }^{\circ} \mathrm{AP}$ is currently a GlaxoSmithKline AG employee.

\section{ABSTRACT}

I n the EMN01 trial, the addition of an alkylator (melphalan or cyclophosphamide) to lenalidomide-steroid induction therapy was prospectively evaluated in transplant-ineligible patients with multiple myeloma. After induction, patients were randomly assigned to maintenance treatment with lenalidomide alone or with prednisone continuously. The analysis presented here (median follow-up of 71 months) is focused on maintenance treatment and on subgroup analyses defined according to the International Myeloma Working Group Frailty Score. Of the 654 evaluable patients, 217 were in the lenalidomide-dexamethasone arm, 217 in the melphalan-prednisone-lenalidomide arm and 220 in the cyclophosphamide-prednisone-lenalidomide arm. With regards to the Frailty Score, 284 (43\%) patients were fit, 205 (31\%) were intermediate-fit and $165(25 \%)$ were frail. After induction, 402 patients were eligible for maintenance therapy (lenalidomide arm, $n=204$; lenalidomideprednisone arm, $n=198$ ). After a median duration of maintenance of 22.0 months, progression-free survival from the start of maintenance was 22.2 months with lenalidomide-prednisone vs. 18.6 months with lenalidomide (hazard ratio $0.85, P=0.14$ ), with no differences across frailty subgroups. The most frequent grade $\geq 3$ toxicity was neutropenia $(10 \%$ of lenalidomide-prednisone and $21 \%$ of lenalidomide patients; $P=0.001)$. Grade $\geq 3$ non-hematologic adverse events were rare $(<15 \%)$. In fit patients, melphalan-prednisone-lenalidomide significantly prolonged progression-free survival compared to cyclophosphamide-prednisone-
Ferrata Storti Foundation

Haematologica 2020

Volume 105(7):1937-1947

\section{Correspondence:}

SARA BRINGHEN

sarabringhen@yahoo.com

Received: May 20, 2019.

Accepted: September 26, 2019.

Pre-published: October 3, 2019.

doi:10.3324/haematol.2019.226407

Check the online version for the most updated information on this article, online supplements, and information on authorship \& disclosures: www.haematologica.org/content/105/7/1937

(C)2020 Ferrata Storti Foundation

Material published in Haematologica is covered by copyright. All rights are reserved to the Ferrata Storti Foundation. Use of published material is allowed under the following terms and conditions:

https://creativecommons.org/licenses/by-nc/4.0/legalcode. Copies of published material are allowed for personal or internal use. Sharing published material for non-commercial purposes is subject to the following conditions: https://creativecommons.org/licenses/by-nc/4.0/legalcode, sect. 3. Reproducing and sharing published material for commercial purposes is not allowed without permission in writing from the publisher. 
lenalidomide (hazard ratio $0.72, P=0.05$ ) and lenalidomide-dexamethasone (hazard ratio $0.72, P=0.04$ ). Likewise, a trend towards a better overall survival was noted for patients treated with melphalan-prednisone-lenalidomide or cyclophosphamide-prednisone-lenalidomide, as compared to lenalidomide-dexamethasone. No differences were observed in intermediate-fit and frail patients. This analysis showed positive outcomes of maintenance with lenalidomide-based regimens, with a good safety profile. For the first time, we showed that fit patients benefit from a full-dose triplet regimen, while intermediate-fit and frail patients benefit from gentler regimens. ClinicalTrials.gov registration number: NCT01093196.

\section{Introduction}

In the last decade, the increased use of novel agents as initial therapy for multiple myeloma (MM) significantly improved overall survival (OS) in patients ineligible for autologous stem-cell transplantation (ASCT). ${ }^{1}$ In Europe, two triplet regimens - bortezomib-melphalan-prednisone and melphalan-prednisone-thalidomide - are considered standards of care for elderly patients ineligible for ASCT ${ }^{2,3}$ Recently, based on the results of the MM020 trial, a new doublet regimen with no alkylating agent was introduced as a new standard for the treatment of transplant-ineligible patients with newly diagnosed MM. That study prospectively compared outcomes of patients treated with melphalan-prednisone-thalidomide vs. lenalidomide and low-dose dexamethasone (Rd), and found that Rd until disease progression improved progression-free survival (PFS) and OS, as compared with melphalan-prednisone-thalidomide. ${ }^{4}$ The phase III trial MM-015 showed that melphalan-prednisonelenalidomide (MPR) followed by maintenance with lenalidomide significantly prolonged PFS, as compared with melphalan-prednisone or MPR without maintenance. ${ }^{5}$

Maintenance therapy with lenalidomide improves outcome and its role has been extensively investigated both in ASCT-eligible and -ineligible patients. A recent metaanalysis of three randomized phase III trials confirmed PFS and OS advantages for lenalidomide maintenance after ASCT vs. placebo or observation. In the MM-015 trial, elderly patients were treated with lenalidomide as induction and maintenance, which reduced the risk of progression by $51 \%$ compared to lenalidomide as induction without maintenance..$^{5}$ In the Myeloma XI trial, lenalidomide maintenance reduced the risk of progression by $56 \%$ in comparison with observation. ${ }^{6}$ Moreover, in this trial both ASCT-eligible and -ineligible patients benefited from lenalidomide maintenance.

The advantage of adding steroids to immunomodulatory drugs during maintenance therapy is unclear. In young patients eligible for ASCT, after a median follow-up of 41 months, median PFS and OS did not differ significantly between patients treated with lenalidomide plus prednisone or lenalidomide alone. No data are available from elderly patients ineligible for ASCT?

The choice of best treatment for each patient is troublesome, especially in elderly patients, since they represent a heterogeneous population in terms of both physical and psycho-social functioning. Furthermore, it is now accepted that chronological and biological ages may not correspond, and that the presence of frailty, comorbidities and disabilities can affect therapy endurance. The OS of frail patients is impaired due to toxic side effects from first-line treatment which may preclude second-line treatment, with third-line therapies in >80-year old MM patients being extremely rare. The "one size fits all" is no longer a suitable approach, and many recommendations suggested that fit patients may benefit from triplet regimens, while intermediate-fit and frail patients may benefit from doublet regimens. ${ }^{8,9}$ There are no data from prospective trials supporting these recommendations and a formal comparison between an alkylator-containing triplet regimen $v s$. an alkylator-free doublet regimen, both including lenalidomide, has not yet been performed.

The EMN01 study was designed to compare the PFS of patients treated with triplet $v s$. doublet induction regimens and the PFS following maintenance treatment with lenalidomide-prednisone vs. lenalidomide alone. Furthermore, before treatment, a geriatric assessment to assess patients' frailty status according to the International Myeloma Working Group (IMWG) Frailty Score was performed. With this analysis, after more than 5 years of follow-up, we would like to report the safety and efficacy of maintenance treatment in our patients and to perform a post-hoc analysis according to frailty status in both induction and maintenance treatment arms.

\section{Methods}

\section{Study design}

The study was conducted in 58 Italian and nine Czech centers between August 2009 and September 2012. The details of this multicenter randomized (1:1:1) phase III trial have already been reported and are updated here after a median follow-up of 71 months for survivors. ${ }^{10}$ Briefly, 662 newly diagnosed (ND)MM patients ineligible for high-dose therapy plus ASCT because of age ( $\geq 65$ years) or coexisting comorbidities were enrolled. The study was approved by the institutional review boards at each of the participating centers and registered at ClinicalTrials.gov (NCT01093196). All patients gave written informed consent before entering the study, which was performed in accordance with the Declaration of Helsinki.

Per protocol, patients were stratified by age ( $\leq 75$ vs. $>75$ years). Based on the recent IMWG geriatric score that stratifies patients according to their frailty status (fit, intermediate-fit, and frail), ${ }^{11}$ a post-hoc analysis including age ( $\leq 75 \mathrm{vs} .76-80 \mathrm{vs}$. $>80$ years), comorbidities (according to the Charlson score) and cognitive/physical status (according to the Activities of Daily Living and the Instrumental Activities of Daily Living scores) was conducted (Online Supplementary Table S1).

\section{Procedures}

Six hundred fifty-four patients were randomly assigned to receive induction (Online Supplementary Figure S1) with nine 28day cycles of $\mathrm{Rd}(\mathrm{n}=217)$ or MPR $(\mathrm{n}=217)$ or cyclophosphamideprednisone-lenalidomide (CPR) $(n=220)$. Rd patients received lenalidomide $25 \mathrm{mg} /$ day for 21 days; dexamethasone $40 \mathrm{mg}$ on days $1,8,15,22$ in patients $65-75$ years old and $20 \mathrm{mg}$ in those $>75$ years of age. MPR patients received lenalidomide $10 \mathrm{mg} /$ day for 
21 days; oral melphalan $0.18 \mathrm{mg} / \mathrm{kg}$ for 4 days in patients 65-75 years old and $0.13 \mathrm{mg} / \mathrm{kg}$ in those $>75$ years of age; prednisone 1.5 $\mathrm{mg} / \mathrm{kg}$ for 4 days. CPR patients received lenalidomide $10 \mathrm{mg} /$ day for 21 days; oral cyclophosphamide $50 \mathrm{mg}$ every other day for 28 days in patients $65-75$ years old and $50 \mathrm{mg}$ every other day for 21 days in those $>75$ years of age; prednisone $25 \mathrm{mg}$ every other day. After induction, patients were randomized to receive maintenance treatment with lenalidomide alone (R) $10 \mathrm{mg}$ on days 1-21 every 28 days, or in combination with prednisone (RP) $25 \mathrm{mg}$ every other day continuously. After the inclusion of the first 120 patients, the protocol was amended to increment the doses of lenalidomide and cyclophosphamide in patients 65-75 years old in the CPR group, due to negligible toxicities in comparison with those in the two other treatment arms. Therefore, the CPR induction schedule was changed to lenalidomide $25 \mathrm{mg}$ /day for 21 days and oral cyclophosphamide $50 \mathrm{mg} /$ day for 21 days.

\section{Statistical analysis}

Updated analyses were performed using data collected on October 31, 2017. All the results were evaluated on an intentionto-treat basis. For univariate analyses, the time-to-event curves were estimated using the Kaplan-Meier method and compared using the log-rank test. Time to event was expressed as median or as 5-year Kaplan-Meier estimate. The Cox proportional hazards model was used to estimate the hazard ratio (HR) values and the $95 \%$ confidence intervals (95\% CI). Data were analyzed as of May 2018 using R (v3.1.1).

\section{Results}

A total of 654 patients were randomly assigned to receive induction with $\mathrm{Rd}(\mathrm{n}=217)$ or $\mathrm{MPR}(\mathrm{n}=217)$ or $\mathrm{CPR}$ $(\mathrm{n}=220)$ (Online Supplementary Figure S1). Baseline demographics and disease characteristics were previously reported $^{10}$ and were well balanced among the three groups. The median age was 73 years in the Rd and CPR arms, and 74 years in the MPR arm. Twenty-five percent of patients were classified as frail and these patients were evenly distributed among the treatment arms. A total of 402 patients completed the assigned induction treatment and were randomly allocated to receive maintenance with RP ( $n=198)$ or $R(n=204)$ (Online Supplementary Figure S1, Table 1 for baseline characteristics).

The median follow-up for survivors was 71 months from enrollment. Progression or death occurred in 177 patients (82\%) in the Rd arm, $166(76 \%)$ in the MPR arm, and $194(88 \%)$ in the CPR arm. The median PFS was 18.6 months with the doublet and 20.8 months with the triplet combinations (HR 1.05, 95\% CI: 0.87-1.25, $P=0.62$ ) (Online Supplementary Figure S2). The median OS was 61.5 months with doublet and 65.7 months with triplet regimens (HR 1.09, 95\% CI: 0.87-1.37, P=0.47). Comparing the three arms separately, the median PFS was 18.6 months in the Rd arm, 22.2 months in the MPR arm and 18.9 months in the CPR arm (MPR vs. CPR: HR 0.78, 95\% CI: 0.63-0.96, $P=0.02$; MPR vs. Rd: HR 0.84, 95\% CI: $0.68-$ $1.04, P=0.11$ ) (Figure $1 \mathrm{~A}$ ). The median time to next treatment (TNT) was 23.8 months in the Rd arm, 28.7 months in the MPR arm and 23.8 months in the CPR arm (MPR vs. CPR: HR 0.79, 95\% CI: 0.64-0.98, $P=0.03$; MPR vs. Rd: HR $0.82,95 \%$ CI: 0.66-1.02, $P=0.07$ ) (Figure $1 \mathrm{~B}$ ). The median progression-free survival 2 (PFS-2) was 41.2 months in the $\mathrm{Rd}$ arm, 40.2 months in the MPR arm and 40.8 months in the CPR arm (MPR vs. CPR: HR 0.90, 95\% CI: 0.72-1.14,
$P=0.40$; MPR vs. Rd: HR 0.94, 95\% CI: 0.74-1.19, $P=0.63)$ (Figure 1C). Death occurred in 115 patients (53\%) in the Rd arm, 108 (50\%) in the MPR arm and 107 (49\%) in the CPR arm. The median OS was 61.5 months with Rd, 65.2 months with MPR and 66.4 months with CPR (MPR vs. CPR: HR 1.03, 95\% CI: 0.79-1.35, $P=0.83$; MPR vs. Rd: HR 0.93, 95\% CI: $0.72-1.22, P=0.61$ ) (Figure 1D). The subgroup analysis of induction treatment in patients with standard- and high-risk cytogenetics showed the same trends observed in the overall population (Online Supplementary Figure S3).

A post-hoc analysis according to patients' frailty was performed (Figure 2). In fit patients, an advantage with the triplet regimen MPR was detected: the median PFS was 21.2 months in patients treated with $\mathrm{Rd}, 25.6$ months in the MPR arm and 21.7 months in patients given CPR (MPR vs. CPR: HR 0.72, 95\% CI: 0.52-1.00, $P=0.05$; MPR vs. Rd: HR 0.72, 95\% CI: 0.52-0.99, $P=0.04$ ) (Figure 2A). The median OS was 50.2 months in the Rd group, shorter than in both the MPR (79.9 months) and CPR groups (82.9 months) (MPR vs. CPR: HR 1.11, 95\% CI: 0.72-1.71, $P=0.65$; MPR vs. Rd: HR 0.75, 95\% CI: 0.50-1.12, $P=0.16$ ) (Figure $2 \mathrm{~B}$ ). In intermediate-fit patients, no advantage of one regimen over the others was found: the median PFS was 16.6 months in the Rd arm, 20 months in the MPR arm and 20.9 months in the CPR arm (Figure 2C). The median OS was not reached in the $\mathrm{Rd}$ arm, was 60.8 months in the MPR arm and was 66.7 months in the CPR arm (Figure 2D). Not even in frail patients was one regimen found to be superior to another: the median PFS was 18.2 months in the Rd arm, 21.5 months in the MPR arm and 13.8 months in the CPR arm (Figure 2E). The median OS was 48.2 months with Rd, 44.7 months with MPR and 40.5 months with CPR (Figure 2F).

Table 1. Demographics and baseline characteristics of the patients receiving maintenance treatment.

\begin{tabular}{lcc}
$\begin{array}{l}\text { Patients' } \\
\text { characteristics }\end{array}$ & $\begin{array}{c}\text { Lenalidomide (Ri) } \\
(\mathrm{n}=204)\end{array}$ & $\begin{array}{c}\text { Lenalidomide- } \\
\text { prednisone (RP) (n=198) }\end{array}$ \\
Age range, years & $50-89$ & $65-87$ \\
Median, years & 73 & 73 \\
$>75$ years, $n(\%)$ & $61(30 \%)$ & $64(32 \%)$ \\
Sex (male), $n(\%)$ & $86(42 \%)$ & $105(53 \%)$ \\
\hline Karnofsky score & $60-100$ & $60-100$ \\
Median & 90 & 90 \\
$<80, \mathrm{n}(\%)$ & $37(18 \%)$ & $43(22 \%)$
\end{tabular}

\begin{tabular}{lll} 
Fitness & & \\
Fit, n (\%) & $101(50 \%)$ & $91(46 \%)$ \\
Intermediate-fit, n (\%) & $63(31 \%)$ & $58(29 \%)$ \\
Frail, n (\%) & $40(20 \%)$ & $49(25 \%)$ \\
\hline
\end{tabular}

\section{Creatinine clearance,}

$\mathrm{mL} / \mathrm{min}$

Median, $\mathrm{mL} / \mathrm{min}$

30-168.9

72

$30-150$

International Staging System score

\begin{tabular}{lll} 
I, n (\%) & $65(32 \%)$ & $65(33 \%)$ \\
II, n (\%) & $92(45 \%)$ & $88(44 \%)$ \\
III, n (\%) & $47(23 \%)$ & $45(23 \%)$ \\
\hline
\end{tabular}

Cytogenetic abnormalities by FISH

Data available, $\mathrm{n}(\%) \quad 163(80 \%) \quad 162(82 \%)$

Data missing, $\mathrm{n}(\%) \quad 41(20 \%) \quad 36(18 \%)$

High risk*, n (\%) $\quad 36(18 \%) \quad 37(19 \%)$

* At least one among deletion $17 \mathrm{p}$ [del(17p)], translocation $(4 ; 14)[\mathrm{t}(4 ; 14)]$ or translocation $(14 ; 16)[\mathrm{t}(14 ; 16)]$. FISH: fluorescence in situ hybridization. 
During maintenance, $31 \%$ of patients in the RP group and $20 \%$ of patients in the $\mathrm{R}$ group had an improvement in their quality of response. In the RP group, the partial response (PR) rate increased from $87 \%$ to $95 \%$, the very good PR (VGPR) rate from $33 \%$ to $58 \%$, and the complete response (CR) rate from $5 \%$ to $9 \%$. In the R group, the PR rate increased from $83 \%$ to $88 \%$, the VGPR rate from $33 \%$ to $47 \%$, and the CR rate from $2 \%$ to $7 \%$.

After a median follow-up of 62 months from the random assignment to maintenance treatment arms, progression or death occurred in 153 patients $(77 \%)$ in the RP group and in 164 (80\%) in the R group. The median PFS was 22.2 months with RP and 18.6 months with R (HR
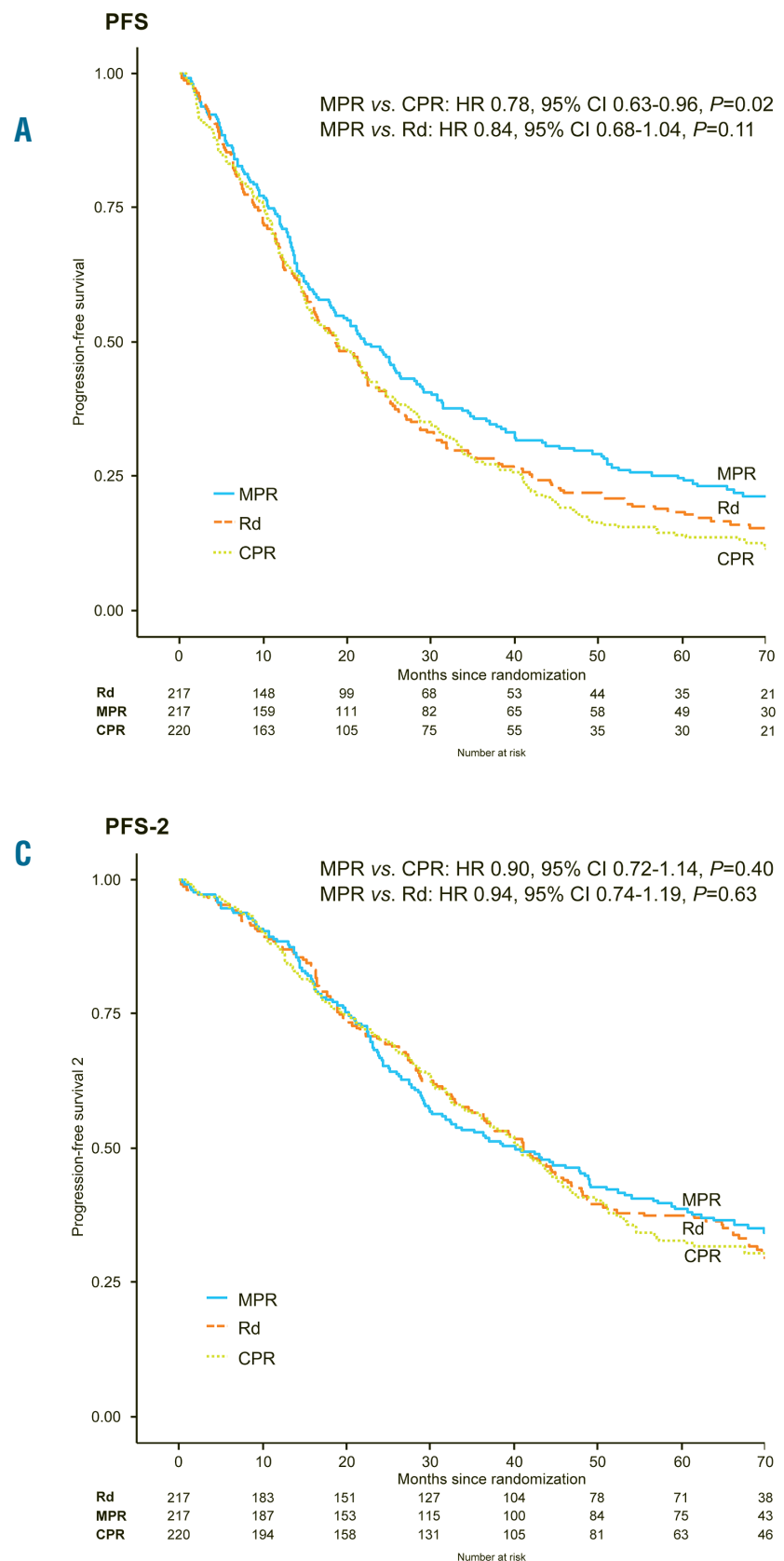

0.85, 95\% CI: 0.68-1.06, $P=0.14$ ) (Figure $3 \mathrm{~A})$. The median TNT was 32.4 months with RP and 29.8 months with $\mathrm{R}$ (HR 0.95, 95\% CI: 0.75-1.20, $P=0.67$ ) (Figure 3B). In both groups, maintenance therapy delayed the median time to next therapy (clinical progression) by approximately 10 months in comparison with the median PFS (biochemical progression). The median PFS-2 was 53.3 months with RP and 42.3 months with R (HR 1.04, 95\% CI: 0.80-1.35, $P=0.79$ ) (Figure 3C). Death occurred in 92 patients (46\%) in the RP group and 78 (38\%) in the R group. The median $\mathrm{OS}$ was 72.3 months with $\mathrm{RP}$ and not reached with $\mathrm{R}$ therapy (HR 1.21, 95\% CI: 0.89-1.64, $P=0.22$ ) (Figure 3D). Subgroup analysis of maintenance treatment in patients
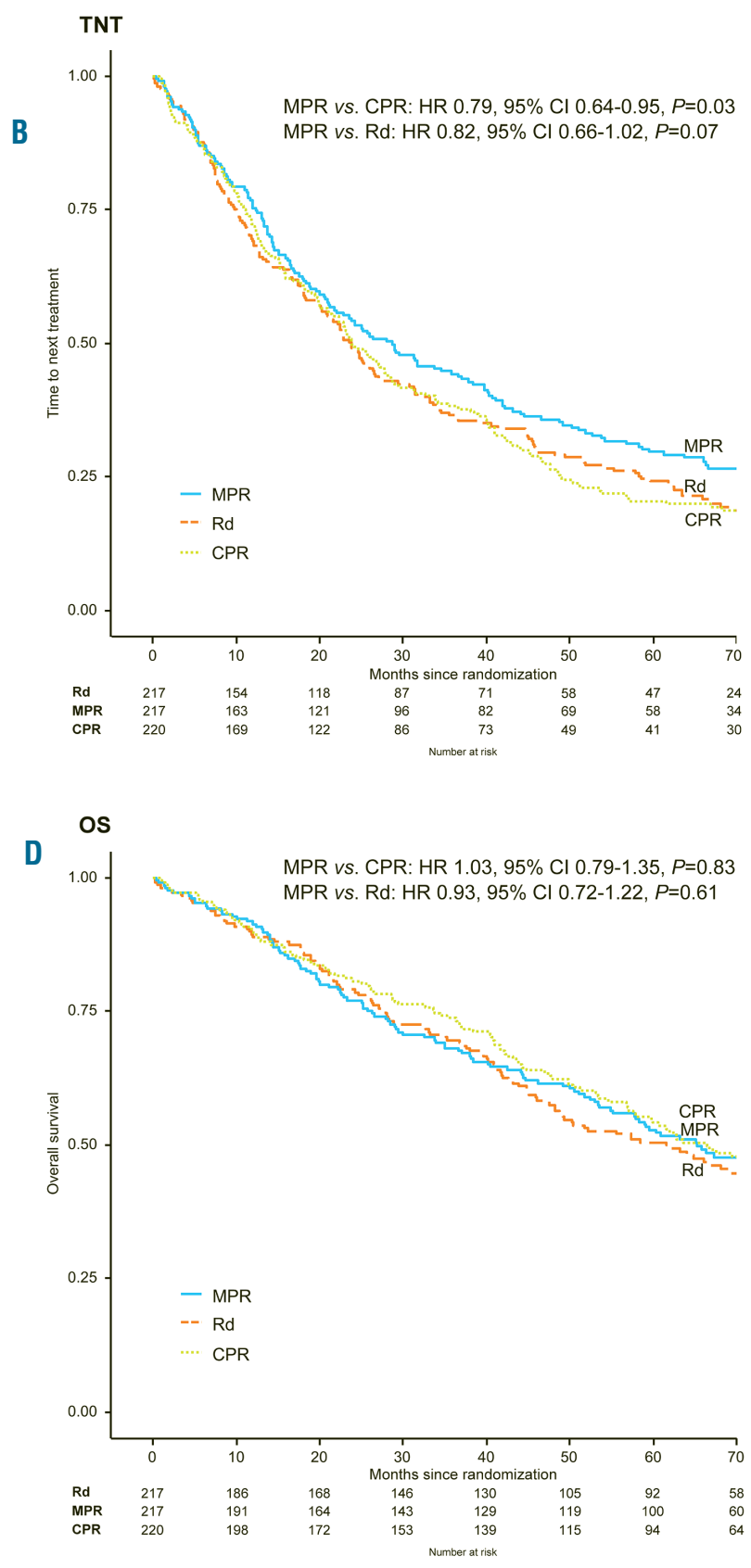

Figure 1. Survival outcomes according to induction treatment arm. (A) Progression-free survival, (B) time to next treatment, (C) progression-free survival 2 and (D) overall survival are shown. All time to events were calculated from the time of random assignment to induction treatment arms. MPR: melphalan-prednisone-lenalidomide; CPR: cyclophosphamide-prednisone-lenalidomide; Rd: lenalidomide-dexamethasone; PFS: progression-free survival; PFS-2: progression-free survival 2; TNT: time to next treatment; OS: overall survival; HR: hazard ratio; Cl: confidence interval; $P$ : $P$ value. 
A

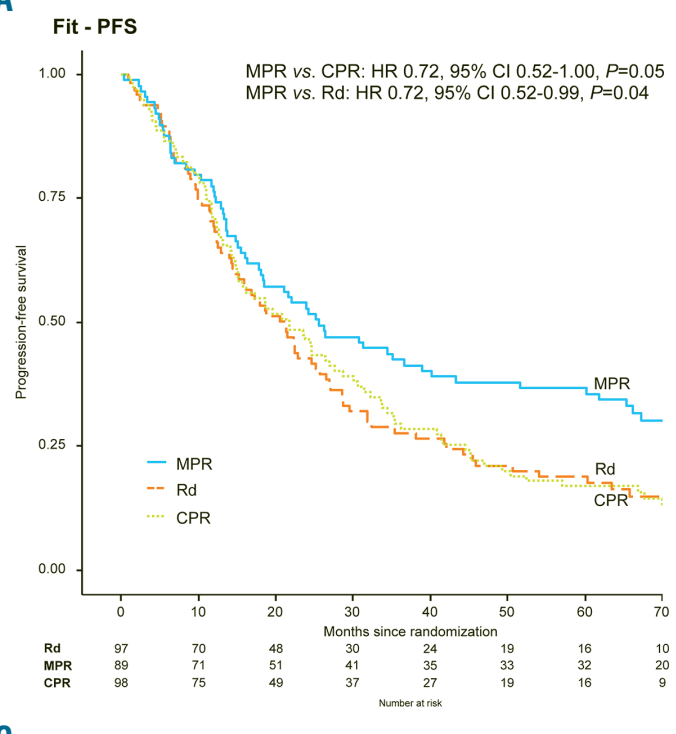

C

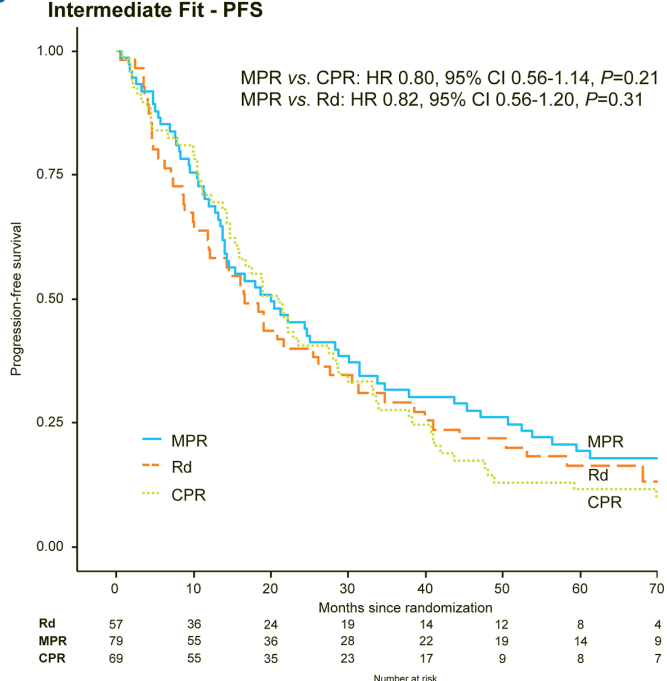

E

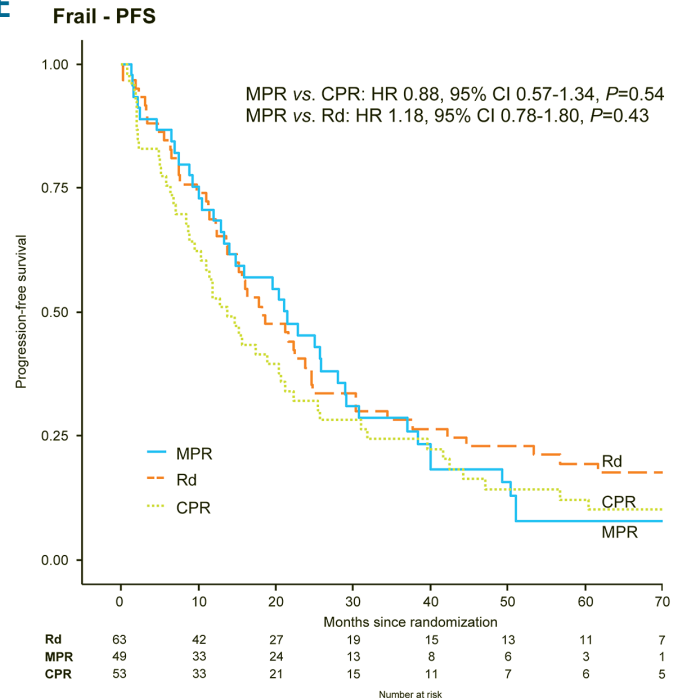

B

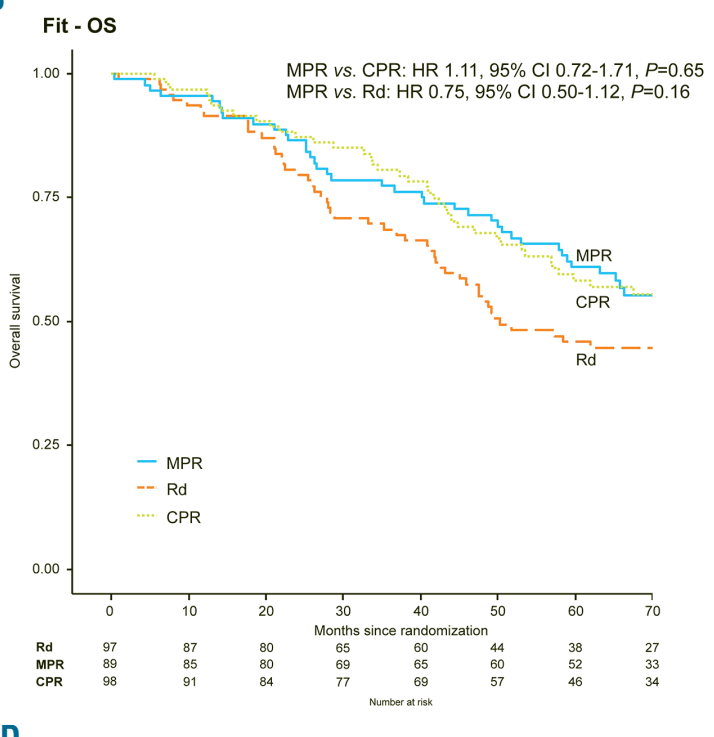

D

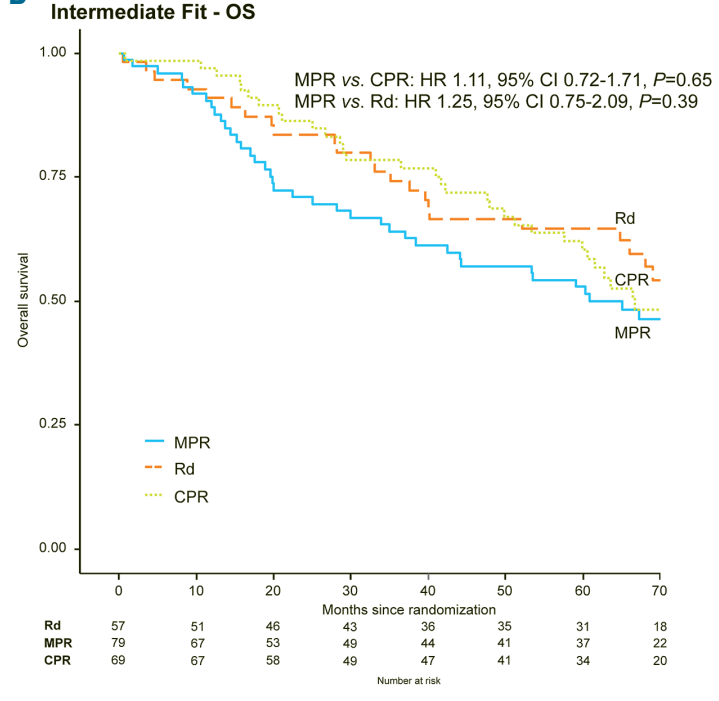

F Frail-os

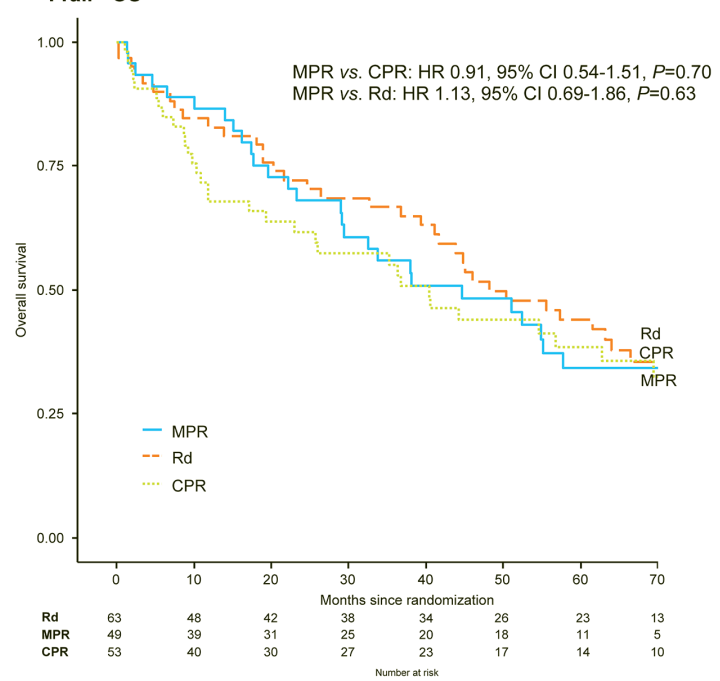

Figure 2. Post-hoc analysis according to frailty status in patients treated with different induction treatments. (A, B) Progression-free survival (PFS) (A) and overall survival (OS) (B) in fit patients according to treatment arm. (C, D) PFS (C) and OS (D) in intermediate-fit patients according to treatment arm. (E; F) PFS (E) and OS $(F)$ in frail patients according to treatment arm. All time to events were calculated from the time of random assignment to induction treatment arms. MPR: melphalan-prednisone-lenalidomide; CPR: cyclophosphamide-prednisone-lenalidomide; Rd: lenalidomide-dexamethasone; HR: hazard ratio; Cl: confidence interval; P: $P$ value. 
A

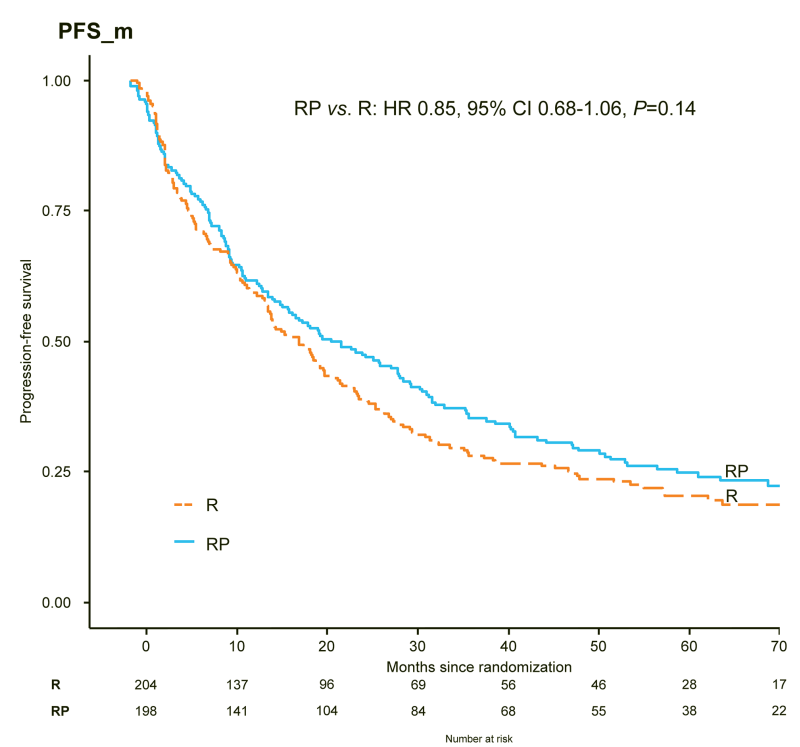

C

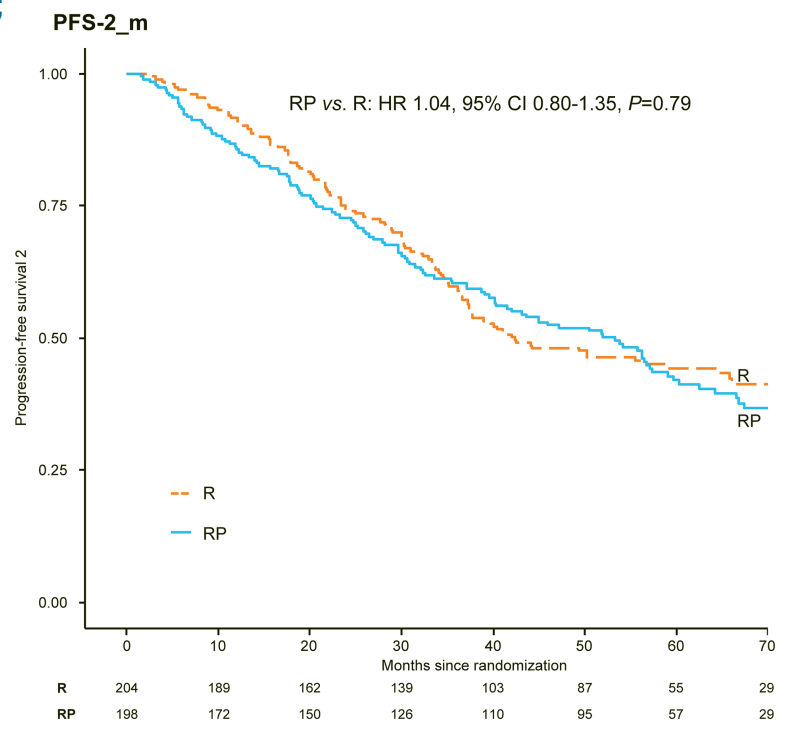

B

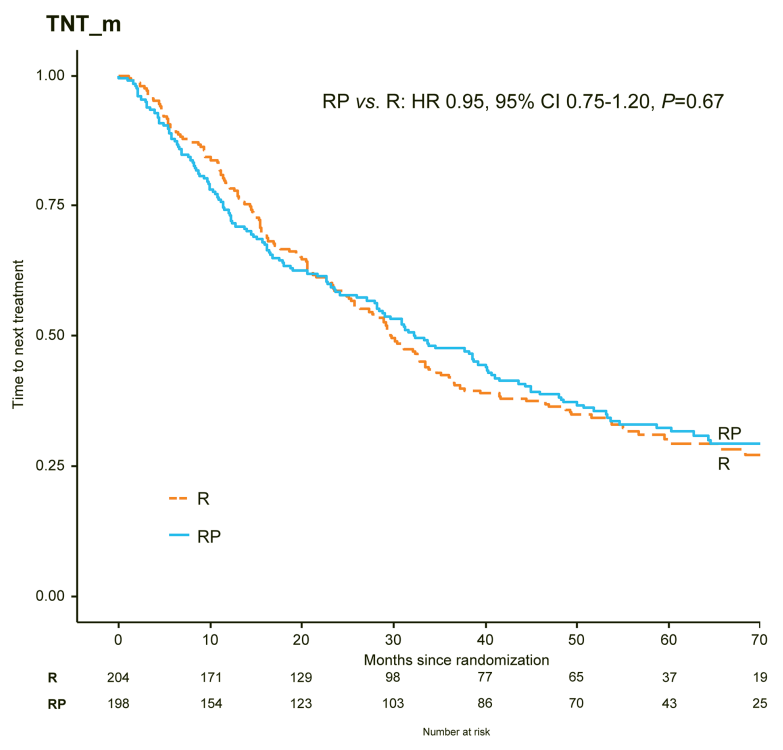

D

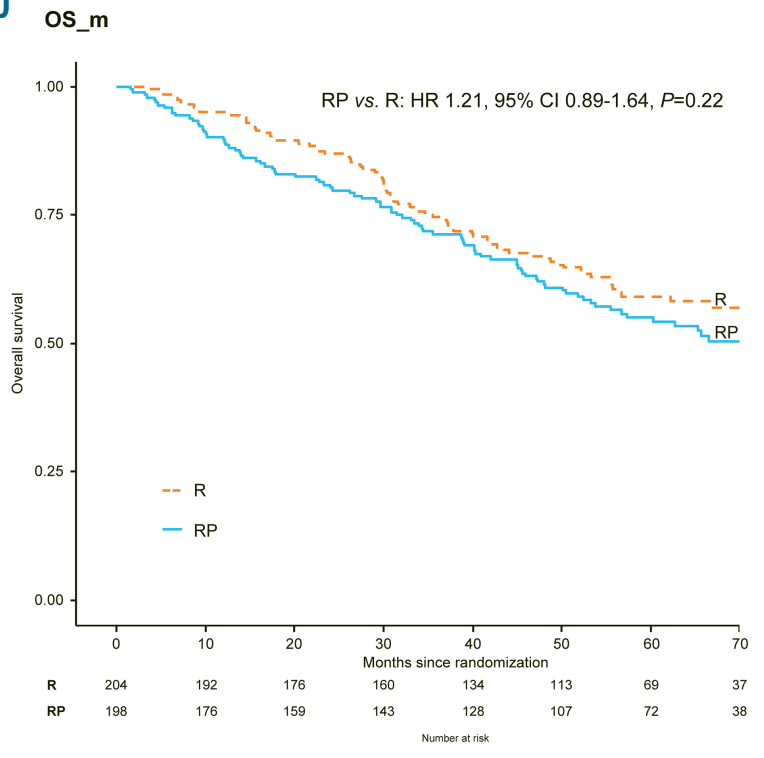

Figure 3. Survival outcomes according to maintenance treatment arm. (A) Progression-free survival, (B) time to next treatment, (C) progression-free survival 2 and (D) overall survival. All time to events were calculated from the time of random assignment to maintenance treatment arms (_m). R: lenalidomide; RP: lenalidomideprednisone; PFS: progression-free survival; PFS-2: progression-free survival 2; TNT: time to next treatment; OS: overall survival; _m: from the random assignment to maintenance treatment arms; HR: hazard ratio; $\mathrm{Cl}$ : confidence interval; $P$ : $P$ value.

with standard- or high-risk cytogenetics showed the same trends observed in the overall population (Online Supplementary Figure S4).

A post-hoc analysis according to patients' frailty was also performed for the maintenance phase (Figure 4) and no significant advantage of one regimen over the other was found. In fit patients, the median PFS from start of maintenance was 24.4 months with $\mathrm{RP}$ and 19.6 months with R (HR 0.84, 95\% CI: 0.60-1.16, $P=0.29$ ) (Figure 4A). Not even in intermediate-fit and frail patients was one regimen found to be superior to the other (Figure 4C, E). No difference in $\mathrm{OS}$ was detected (Figure 4B, D, F).

Safety profiles of induction were reported in the initial analysis..$^{10}$ Briefly, the most frequent grade $\geq 3$ toxicities were hematologic. At least one grade $\geq 3$ hematologic adverse event was reported in $29 \%$ of patients treated with $\mathrm{Rd}, 68 \%$ of those treated with MPR and $32 \%$ of patients treated with CPR $(P<0.001)$. The rate of at least one grade $\geq 3$ non-hematologic adverse event did not exceed $31 \%$ in any of the three arms. The most frequent grade $\geq 3$ non-hematologic toxicities were infections $(9 \%$ with $\mathrm{Rd}, 11 \%$ with MPR and $6.5 \%$ with CPR), constitutional adverse events (5\% with $\mathrm{Rd}, 9.5 \%$ with MPR and $3.5 \%$ with CPR) and cardiac toxicities (6\% with Rd, $4.5 \%$ with MPR and 6\% with CPR); no significant differences were detected among the three arms. The rate of discontinuation due to adverse events was similar in the three arms: $14 \%$ in the $\mathrm{Rd}$ arm, $18 \%$ in the MPR arm and $15 \%$ in the CPR arm. Lenalidomide was reduced in $16 \%$ of patients treated with $\mathrm{Rd}, 21 \%$ of those treated with MPR and $18 \%$ of CPR-treated patients, without significant differences among the three arms. 
Lenalidomide-based regimens in elderly MM

A

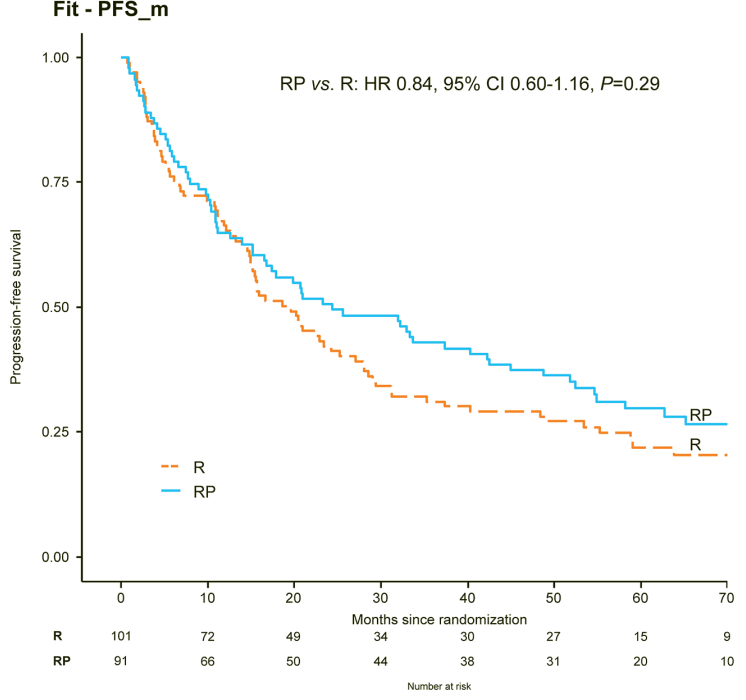

C Intermediate Fit - PFS_m
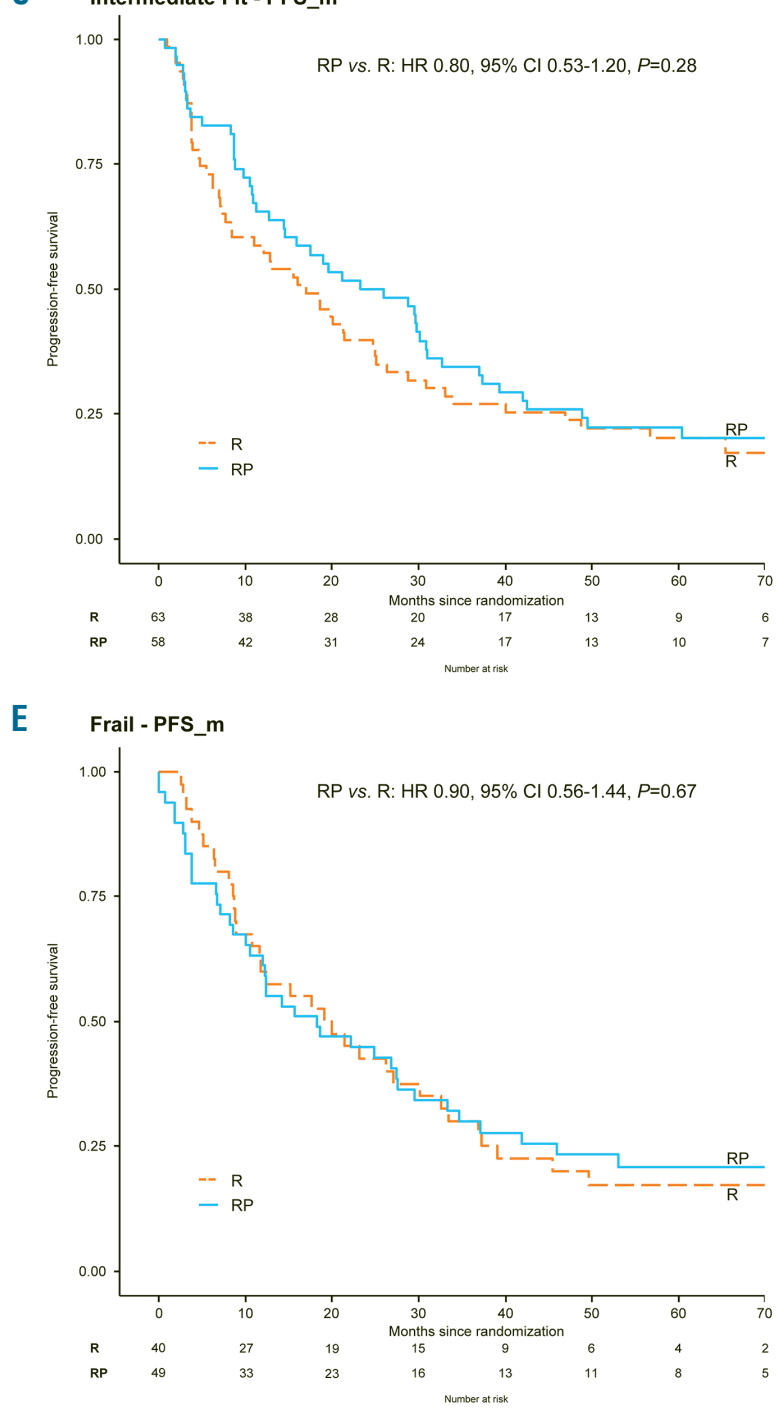

B Fit-os_m

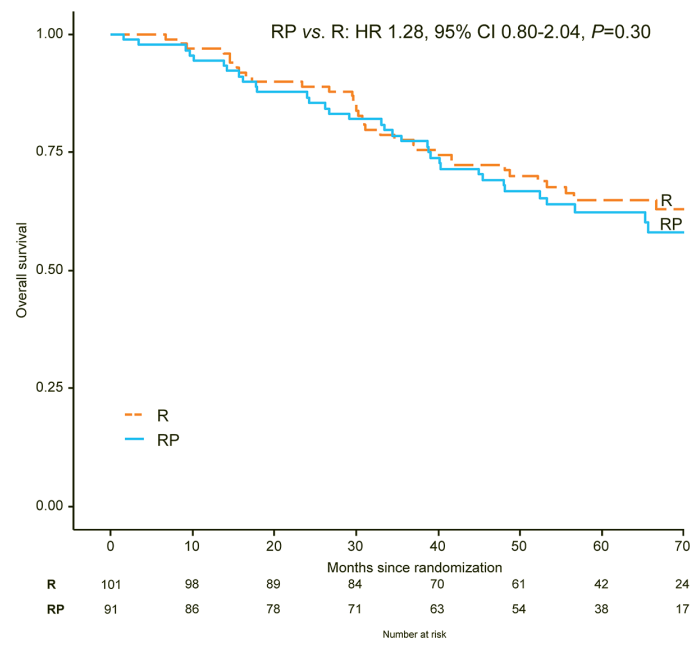

D Intermediate Fit - os_m
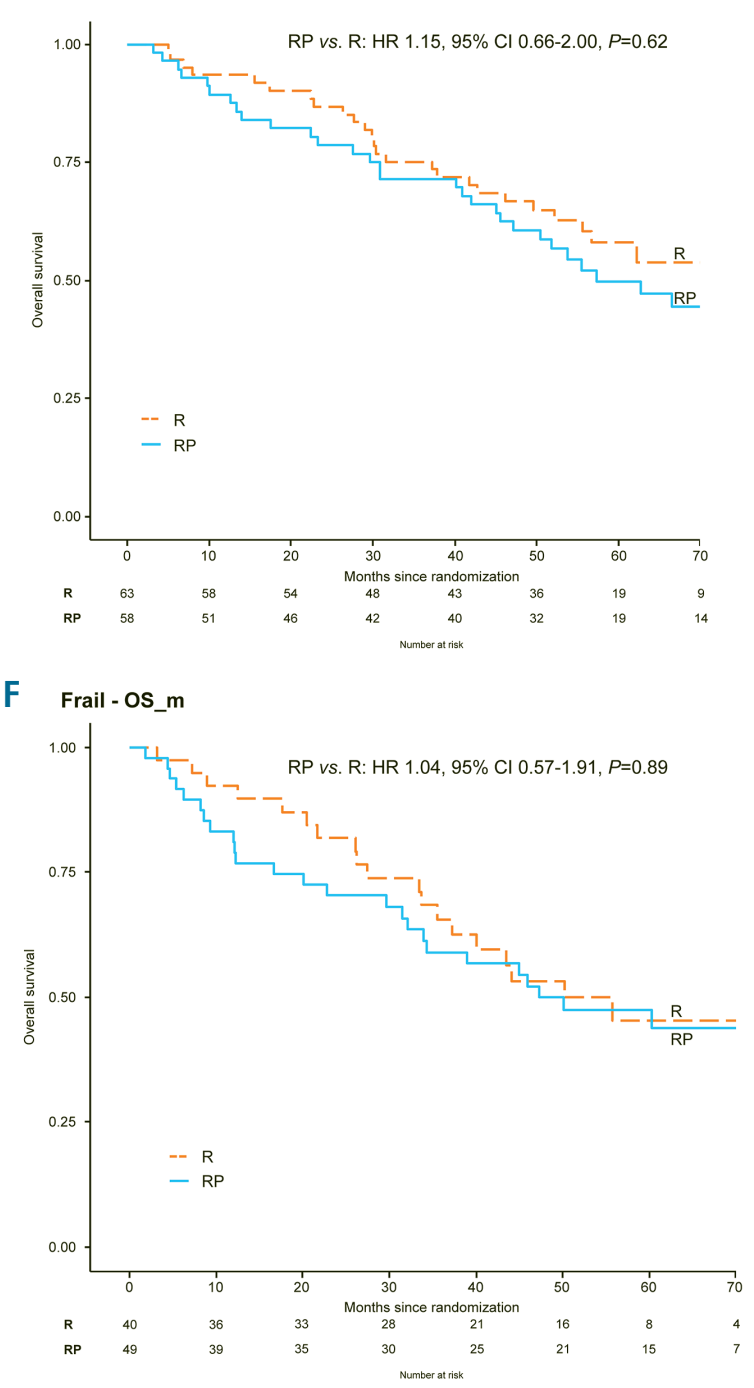

Figure 4. Post-hoc analysis according to frailty status in patients treated with different maintenance treatments. (A, B) Progression-free survival (PFS) (A) and overall survival (OS) (B) in fit patients according to treatment arm. (C, D) PFS (C) and OS (D) in intermediate-fit patients according to treatment arm. (E, F) PFS (E) and OS $(F)$ in frail patients according to treatment arm. All time to events were calculated from the time of random assignment to maintenance treatment arms (_m). R: Ienalidomide; RP: Ienalidomide-prednisone; PFS_m: progression-free survival from the random assignment to maintenance treatment arms; OS_m: overall survival from the random assignment to maintenance treatment arms; HR: hazard ratio; $\mathrm{Cl}$ : confidence interval; $P: P$ value.

haematological | 2020; 105(7)

1943 
The incidence of at least one hematologic adverse event was similar in fit, intermediate-fit and frail patients. The rate of non-hematologic adverse events as well as the rate of discontinuation due to adverse events increased with worsening of fitness status (Online Supplementary Table S2). Data from each induction treatment group are presented in Table 3. Frail patients receiving the alkylatingcontaining regimens had the highest rate of discontinuation due to adverse events.

During maintenance, the most frequent grade $\geq 3$ toxicity was neutropenia, which occurred in $10 \%$ of RP and $21 \%$ of $\mathrm{R}$ patients $(P=0.001)$ (Table 2$)$. Grade $\geq 3$ nonhematologic adverse events were rare and occurred in $<15 \%$ of patients. The proportion of patients requiring dose discontinuation due to adverse events during maintenance was $18 \%$ in the $\mathrm{R}$ arm and $21 \%$ in the RP arm. The proportion of patients requiring dose reduction during maintenance was $9 \%$ in the RP arm and $16 \%$ in the $\mathrm{R}$ arm $(P=0.05)$. Fifteen cases of second primary malignancies were recorded: six (3\%) in the RP group and nine $(4 \%)$ in the $\mathrm{R}$ group. All second primary malignancies were solid tumors.

In the RP group, 133 patients required a second line of therapy: 94 (71\%) received bortezomib, $3(2 \%)$ thalidomide or lenalidomide, 17 (13\%) other chemotherapy, 16 $(12 \%)$ died before starting treatment and $3(2 \%)$ were lost to follow-up. In the R group, 138 patients required a second line of therapy: 102 (74\%) received bortezomib, 3 $(2 \%)$ thalidomide or lenalidomide, 21 (15\%) other chemotherapy, 7 (5\%) died before starting treatment and $5(4 \%)$ were lost to follow-up.

The incidences of at least one hematologic and nonhematologic grade $\geq 3$ adverse events were similar in fit, intermediate-fit and frail patients. Frail patients had the highest rate of discontinuation due to adverse events; a trend towards a higher discontinuation due to adverse events was found in frail vs. fit patients (Online Supplementary Table S2). Data in each maintenance treatment group are presented in Table 4.

\section{Discussion}

One of the aims of this analysis was to compare RP vs. $\mathrm{R}$ alone as maintenance treatment after induction therapy.

While the use of maintenance therapy is a standard approach in young ASCT-eligible patients with newly diagnosed $\mathrm{MM}$, its use in elderly MM patients after induction treatment is more debated.
In our trial, both maintenance regimens improved the quality of response and produced a time from biochemical to clinical relapse of approximately 10 months. Indeed, as recently described, even when neoplastic plasma cells become lenalidomide-refractory, the immunomodulatory effect of lenalidomide on immune cells may help prolong disease control. ${ }^{12}$ A trend toward a slight improvement of PFS in the RP arm was noted as compared to $\mathrm{R}$ alone, while OS data were still immature after only 170 deaths (42\% of patients).

Regarding safety, maintenance treatment with both regimens was feasible with grade $\geq 3$ non-hematologic adverse event rates of less than $15 \%$. The only difference

Table 2. Grade $\geq 3$ adverse events during maintenance treatment.

Grade $\geq 3$ adverse events Lenalidomide (R) Lenalidomide-prednisone
$(n=204)$

\section{Hematologic}

\begin{tabular}{lcc} 
At least one event & $46(23 \%)$ & $26(13 \%)$ \\
Anemia & $4(2 \%)$ & $4(2 \%)$ \\
\hline Neutropenia & $43(21 \%)$ & $19(10 \%)$ \\
Thrombocytopenia & $4(2 \%)$ & $5(3 \%)$ \\
\hline
\end{tabular}

\section{Non-hematologic}

At least one event

\begin{tabular}{lcc} 
At least one event & $22(11 \%)$ & $28(14 \%)$ \\
\hline Cardiological & $2(1 \%)$ & 1 \\
Acute myocardial infarction & 1 & 1 \\
Other & 1 & 0 \\
Vascular & $5(2 \%)$ & $4(2 \%)$ \\
Deep vein thrombosis/ & $2(1 \%)$ & $3(2 \%)$ \\
$\quad$ thromboembolism & & \\
\hline Renal & 1 & $2(1 \%)$ \\
Dermatological & $2(1 \%)$ & $3(2 \%)$ \\
\hline Infectious & $2(1 \%)$ & $4(2 \%)$ \\
Pneumonia & 0 & 1 \\
Bronchitis & 1 & 1 \\
Sepsis & 0 & 1 \\
Other/not specified & 1 & 1 \\
Nervous & $2(1 \%)$ & $7(4 \%)$ \\
\hline Second primary malignancies & $9(4 \%)$ & $6(3 \%)$ \\
Hematologic & 0 & 0 \\
Solid & $9(4 \%)$ & $6(3 \%)$ \\
Other & $9(4 \%)$ & $5(3 \%)$ \\
\hline Discontinuation due & $36(18 \%)$ & $41(21 \%)$ \\
to adverse events & &
\end{tabular}

to adverse events

Table 3. Grade $\geq 3$ hematologic adverse events, non-hematologic adverse events, treatment discontinuation due to adverse events and toxic deaths during induction treatment according to patients' frailty status.

\begin{tabular}{|c|c|c|c|c|c|c|c|c|c|}
\hline Treatment arm (n) & & $\begin{array}{c}C P R \\
(n=220)\end{array}$ & & & $\begin{array}{c}\text { MPR } \\
(n=211)\end{array}$ & & & $\begin{array}{c}\text { Rd } \\
(n=212)\end{array}$ & \\
\hline Frailty Score class (n) & $\begin{array}{l}\text { Fit } \\
(98)\end{array}$ & $\begin{array}{c}\text { Intermediate } \\
\text {-fit (69) }\end{array}$ & $\begin{array}{l}\text { Frail } \\
(53)\end{array}$ & $\begin{array}{l}\text { Fit } \\
(88)\end{array}$ & $\begin{array}{l}\text { Intermediate- } \\
\text { fit (76) }\end{array}$ & $\begin{array}{l}\text { Frail } \\
(47)\end{array}$ & $\begin{array}{l}\text { Fit } \\
(94)\end{array}$ & $\begin{array}{l}\text { Intermediate- } \\
\text { fit (57) }\end{array}$ & $\begin{array}{l}\text { Frail } \\
(61)\end{array}$ \\
\hline Hematologic $\mathrm{AE} \mathrm{G} \mathrm{\geq 3,} \mathrm{n} \mathrm{( \% )}$ & $31(32)$ & $23(33)$ & $17(32)$ & $62(70)$ & $46(61)$ & $35(74)$ & $26(28)$ & $13(23)$ & $22(36)$ \\
\hline Non-hematologic $\mathrm{AE} \mathrm{G} \geq 3$, n (\%) & $22(22)$ & $22(32)$ & $22(42)$ & $22(25)$ & $22(29)$ & $22(45)$ & $24(26)$ & $15(26)$ & $24(39)$ \\
\hline Discontinuation due to $\mathrm{AE}, \mathrm{n}(\%)$ & $8(8)$ & $9(13)$ & $16(30)$ & $10(11)$ & $17(22)$ & $10(21)$ & $9(10)$ & $9(16)$ & $12(20)$ \\
\hline Death due to $\mathrm{AE}, \mathrm{n}(\%)$ & $1(1)$ & 0 & $7(13)$ & $1(1)$ & $2(3)$ & $2(4)$ & $1(1)$ & $1(2)$ & $4(7)$ \\
\hline
\end{tabular}


between treatment arms was the most frequent grade $\geq 3$ neutropenia in the $\mathrm{R}$ arm compared to $\mathrm{RP}$, which did not translate into a higher infection rate. One of the possible explanations of this finding is based on the effect of prednisone on neutrophils. ${ }^{13}$ Glucocorticoid therapy inhibits Lselectin expression on neutrophils that are more prone to enter the bloodstream, delays the migration of circulating neutrophils into tissues, produces a direct antiapoptotic effect on these cells and prompts the release of young neutrophils from the bone marrow leading to an increased peripheral blood neutrophil count.

While dose discontinuation was not different in the RP and $\mathrm{R}$ arms, patients treated with $\mathrm{R}$ alone more often required dose reductions, which slightly impaired the efficacy of maintenance treatment.

The treatment goal in elderly MM patients is not a trivial issue and international guidelines suggesting personalized treatment according to patients' frailty status are currently based on expert opinions, without the availability of highquality evidence., With all the limitations of a post-hoc analysis, this is the first analysis to show that fit patients benefit from a full-dose triplet regimen while intermediatefit and frail patients benefit from gentler regimens.

Indeed, fit patients treated with MPR induction showed a statistically significant PFS advantage over those treated with CPR or Rd. Intermediate-fit and frail patients did not show any PFS benefit from one regimen over the others.

A higher hematologic adverse event rate was noted with MPR induction compared to induction with the other regimens.

After the protocol amendment, introduced because of negligible adverse events, the dose of cyclophosphamide was increased. Nevertheless, despite the amendment, cyclophosphamide could still have been underdosed as compared to the dose delivered in other cyclophosphamide-containing regimens used in younger patients. ${ }^{14,15}$ This issue could have mitigated the impact on PFS of the addition of cyclophosphamide to lenalidomide-steroid doublets even in fit patients.

Hematologic adverse events were not dependent on patients' frailty status, while the rate of non-hematologic adverse events was correlated to the fitness status but not to the type of induction regimen. Indeed, physicians' limitations to treat frail patients effectively are based on the patients' reduced organ function reserve leading to a higher rate of non-hematologic toxicity, rather than on the hematologic toxicity that is mainly dependent on the treatment itself, as observed with the melphalan-containing regimen in this study.
As expected, frail patients experienced the highest discontinuation rate due to toxicity, and discontinuation was more frequent among patients receiving the alkylator-containing regimens than among those treated with the $\mathrm{Rd}$ doublet. It is, therefore, reasonable to support the choice of a full-dose alkylator-containing triplet regimen in fit patients, in order to prioritize the efficacy. Conversely, intermediate-fit and frail patients could benefit from a gentler regimen, since a better safety profile should be pursued in the absence of an advantage in PFS or OS.

In the FIRST trial, a retrospective proxy algorithm (which calculated data from questionnaires on medical history and quality of life) was used to estimate the IMWG Frailty Score. ${ }^{16}$ In that post-hoc analysis, continuous $\mathrm{Rd}$ was compared to an alkylator-containing triplet regimen; however, differently from our analysis, the novel agent used in the control arm was the first-generation immunomodulatory drug thalidomide. Indeed, in that analysis continuous $\mathrm{Rd}$ produced longer PFS and OS compared to melphalan-prednisone-thalidomide across all frailty subgroups, with the greatest benefits observed in fit patients. No lenalidomide-containing triplet regimens were included in that trial.

Despite the limitations of inter-trial comparisons, $\mathrm{Rd}$ induction produced a shorter PFS in our study than in the FIRST study (26 months with continuous $\mathrm{Rd}$ and 21 months with Rd given for 18 months). ${ }^{17}$ Of note, in that trial $\mathrm{Rd}$ was given for a fixed duration of 18 months or continuously, while in our trial Rd was given only for 9 months as induction treatment and then both maintenance regimens included lenalidomide given at a lower dose (10 mg instead of $25 \mathrm{mg}$ ) and lower steroid doses or no steroids at all, depending on the treatment arm. The same observation, with the same limitations of inter-trial comparisons, can also be applied to the control arm of the more recent MAIA study, in which a median PFS of 31.9 months was obtained with continuous $\mathrm{Rd}$ (even longer than the PFS obtained in the FIRST study with an identical regimen). ${ }^{18}$

However, the role of continuous, full-dose treatment vs. full-dose induction followed by low-dose maintenance in frailty-defined populations of elderly patients remains an open issue.

Recently, initial results from a randomized phase III trial comparing continuous $\mathrm{Rd} v \mathrm{~s}$. $\mathrm{Rd}$ induction followed by lenalidomide maintenance (Rd-R) in intermediate-fit patients were reported. ${ }^{19}$ Notably, in this population of patients, continuous Rd did not produce an advantage in PFS compared to $\mathrm{Rd}-\mathrm{R}$ (15.5 months vs. 18.3 months). On the

Table 4. Grade $\geq 3$ hematologic adverse events, non-hematologic adverse events, treatment discontinuation and toxic deaths during maintenance treatment according to patients' frailty status.

\begin{tabular}{|c|c|c|c|c|c|c|}
\hline \multirow{2}{*}{$\begin{array}{l}\text { Treatment arm (n) } \\
\text { Frailty Score class (n) }\end{array}$} & \multicolumn{3}{|c|}{$\begin{array}{l}\text { Lenalidomide (R) } \\
\text { (n=204) }\end{array}$} & \multicolumn{3}{|c|}{$\begin{array}{l}\text { Lenalidomide-prednisone (RP) } \\
\qquad(\mathrm{n}=198)\end{array}$} \\
\hline & $\begin{array}{c}\text { Fit } \\
(101)\end{array}$ & $\begin{array}{l}\text { Intermediate-fit } \\
(63)\end{array}$ & $\begin{array}{l}\text { Frail } \\
(40)\end{array}$ & $\begin{array}{l}\text { Fit } \\
(91)\end{array}$ & $\begin{array}{l}\text { Intermediate-fit } \\
\text { (58) }\end{array}$ & $\begin{array}{l}\text { Frail } \\
(49)\end{array}$ \\
\hline Hematologic AE G z3, n (\%) & $24(24)$ & $13(21)$ & $9(22)$ & $10(11)$ & $6(10)$ & $10(20)$ \\
\hline Non-hematologic $\mathrm{AE} \mathrm{G} \geq 3$, n (\%) & $12(12)$ & $5(8)$ & $5(12)$ & $12(13)$ & $9(16)$ & $7(14)$ \\
\hline Discontinuation due to $\mathrm{AE}, \mathrm{n}(\%)$ & $16(16)$ & $9(14)$ & $11(28)$ & $14(15)$ & $16(28)$ & $11(22)$ \\
\hline Death due to AE, n (\%) & $2(2)$ & $1(2)$ & $2(5)$ & $2(2)$ & $2(3)$ & $4(8)$ \\
\hline
\end{tabular}

AE: adverse events; G: grade; n: number; \%: percentage. 
other hand, event-free survival (progression or death from any cause/lenalidomide discontinuation/hematologic grade 4 or non-hematologic grade 3-4 adverse events) was significantly better in the Rd-R arm than in the continuous Rd arm.

These results suggest that at least in intermediate-fit elderly patients with NDMM, treatment intensity during the maintenance phase can be de-escalated with no negative impact on outcome.

The EMN01 trial enrolled patients from 2009 to 2012; thereafter, less toxic and more effective combinations began to be available. For instance, the addition of bortezomib to Rd led to a clinically meaningful improvement in PFS and OS in NDMM patients without intent for immediate ASCT, irrespective of the patients' age. ${ }^{20}$ In NDMM patients, the addition of the anti-CD38 monoclonal antibody daratumumab to bortezomib-melphalan-prednisone or Rd doubled PFS, with mild and manageable toxicity. ${ }^{18,21}$ Besides, studies exploring the addition of a monoclonal antibody to the bortezomib-Rd combination are ongoing with very promising early results. ${ }^{22}$

Contextualizing our data, quadruplet or triplet regimens containing monoclonal antibodies, immunomodulatory drugs and/or proteasome inhibitors may be the best choices for fit, elderly patients. However, there is still the need for safety and efficacy data on selected intermediate-fit and frail populations receiving new combinations at full or reduced doses. As an example, in the MAIA trial, continuous daratumumab-Rd significantly prolonged PFS compared to continuous Rd, but a higher incidence of infections and a lower lenalidomide cumulative dose due to dose reduction/discontinuation were noted in the daratumumab-Rd arm..$^{23}$ Regarding maintenance, data about combinations of monoclonal antibodies plus immunomodulatory drugs outside of the context of continuous therapy are not currently available in elderly patients. In the experimental arm of the ALCYONE trial, daratumumab monotherapy after induction therapy with the daratumumab-bortezomib-melphalan-prednisone quadruplet was well tolerated and improved the duration and depth of response. ${ }^{24} \mathrm{~A}$ longer follow-up of these two studies will inform us about the safety of monoclonal antibody maintenance and the feasibility of long-term treatment with monoclonal antibodies plus immunomodulatory drugs in elderly patients.

Our analysis has some limitations. The trial was designed to show superiority of a three-drug induction regimen over a two-drug induction regimen in the overall population, and thus the study power was not enough to detect a statistically significant difference in the frailty subgroups. However, the outcome differences between treatment arms in fit patients were high enough to reach significant levels.

Moreover, our analysis based on the frailty status was not pre-specified, but the geriatric assessment adopted to calculate the IMWG Frailty Score was obtained from the enrolled patients before the start of therapy. Although patients' allocation to treatment arms was not stratified by IMWG Frailty Score, the stratification by age led to a uniform distribution of fit, intermediate-fit, and frail patients across treatment arms.

In conclusion, this trial showed that the triplet MPR prolonged PFS compared to gentler regimens in elderly fit but not in intermediate-fit and frail MM patients. In intermediate-fit and frail patients, in the absence of differences in terms of efficacy, safety must be prioritized. Maintenance with lenalidomide-based regimens led to good outcomes with a good safety profile.

These data provide the basis for personalized treatment according to a patients' frailty status. Different combinations of new-generation immunomodulatory drugs, proteasome inhibitors and monoclonal antibodies should be evaluated in fit, intermediate-fit and frail patients to confirm these observations. Novel compounds with a good safety profile in combination with a lenalidomide-based maintenance treatment should also be explored in elderly patients.

\section{Role of the funding source}

The EMNO1 study (NCT01093196) was sponsored by Fondazione Neoplasie Sangue [FO.NE.SA.] ONLUS (Italy) and supported by funding from Celgene, which had no role in the study's design, data collection, data analysis, data interpretation, writing of the report or publication of this contribution. The corresponding author had full access to all the data in the EMNO1 study and had final responsibility for the decision to prepare and submit this manuscript for publication, together with the other authors.

\section{Acknowledgments}

We thank all the patients who participated in the study, the nurses Silvia Boscolo and Concetta Calicchio and the data managers Antonella Fiorillo and Elena Tigano.

\section{References}

1. Palumbo A, Anderson K. Multiple myeloma. N Engl J Med. 2011;364(11):1046-1060.

2. Palumbo A, Bringhen S, Caravita $T$, et al. Oral melphalan and prednisone chemotherapy plus thalidomide compared with melphalan and prednisone alone in elderly patients with multiple myeloma: randomised controlled trial. Lancet. 2006;367(9513):825-831.

3. San Miguel JF, Schlag R, Khuageva NK, et al. Bortezomib plus melphalan and prednisone for initial treatment of multiple myeloma. N Engl J Med. 2008;359(9):906917

4. Benboubker L, Dimopoulos MA, Dispenzieri A, et al. Lenalidomide and dexamethasone in transplant-ineligible patients with myeloma. N Engl J Med. 2014;371(10):906-917

5. Palumbo A, Hajek R, Delforge $M$, et al. Continuous lenalidomide treatment for newly diagnosed multiple myeloma. N Engl J Med. 2012;366(19):1759-1769.

6. Jackson GH, Davies FE, Pawlyn C, et al. Lenalidomide maintenance versus observation for patients with newly diagnosed multiple myeloma (Myeloma XI): a multicentre, open-label, randomised, phase 3 trial. Lancet Oncol. 2019;20(1):57-73.

7. Gay F, Oliva S, Petrucci MT, et al. Chemotherapy plus lenalidomide versus autologous transplantation, followed by lenalidomide plus prednisone versus lenalidomide maintenance, in patients with multiple myeloma: a randomised, multicentre, phase 3 trial. Lancet Oncol. 2015;16(16):1617-1629.
8. Larocca A, Dold SM, Zweegman S, et al Patient-centered practice in elderly myeloma patients: an overview and consensus from the European Myeloma Network (EMN). Leukemia 2018;32(8):1697-1712.

9. Salvini M, D'Agostino M, Bonello F, Boccadoro M, Bringhen S. Determining treatment intensity in elderly patients with multiple myeloma. Expert Rev Anticancer Ther. 2018;18(9):917-930.

10. Magarotto V, Bringhen S, Offidani M, et al. Triplet vs. doublet lenalidomide-containing regimens for the treatment of elderly patients with newly diagnosed multiple myeloma. Blood. 2016;127(9):1102-1108.

11. Palumbo A, Bringhen S, Mateos M-V, et al. Geriatric assessment predicts survival and toxicities in elderly myeloma patients: an International Myeloma Working Group report. Blood. 2015;125(13):2068-2074. 
12. Franssen LE, Nijhof IS, Bjorklund CC, et al. Lenalidomide combined with low-dose cyclophosphamide and prednisone modulates Ikaros and Aiolos in lymphocytes, resulting in immunostimulatory effects in lenalidomide-refractory multiple myeloma patients. Oncotarget. 2018;9(74):3400934021.

13. Nakagawa M, Terashima T, D'yachkova Y, Bondy GP, Hogg JC, van Eeden SF. Glucocorticoid-induced granulocytosis: contribution of marrow release and demargination of intravascular granulocytes. Circulation. 1998;98(21):2307-2313.

14. Stewart AK, Rajkumar SV, Dimopoulos $\mathrm{MA}$, et al. Carfilzomib, lenalidomide, and dexamethasone for relapsed multiple myeloma. N Engl J Med. 2015;372(2):142152.

15. Jakubowiak AJ, Dytfeld D, Griffith KA, et al. A phase $1 / 2$ study of carfilzomib in combination with lenalidomide and low-dose dexamethasone as a frontline treatment for multiple myeloma. Blood. 2012;120(9): 1801-1809.

16. Facon T, Hulin C, Dimopoulos MA, et al. A frailty scale predicts outcomes of patients with newly diagnosed multiple myeloma who are ineligible for transplant treated with continuous lenalidomide plus lowdose dexamethasone on the FIRST trial.
Blood. 2015;126(23). Abstract \#4239 [ASH 2015 57th Meeting].

17. Facon T, Dimopoulos MA, Dispenzieri A, et al. Final analysis of survival outcomes in the phase 3 FIRST trial of up-front treatment for multiple myeloma. Blood. 2018;131(3):301-310

18. Facon T, Kumar SK, Plesner T, et al. Phase 3 randomized study of daratumumab plus lenalidomide and dexamethasone (D-Rd) versus lenalidomide and dexamethasone $(\mathrm{Rd})$ in patients with newly diagnosed multiple myeloma (NDMM) ineligible for transplant (MAIA). Blood. 2018;132(Suppl 1). Abstract \#LBA-2 [ASH 2018 60th Meeting].

19. Larocca A, Salvini M, De Paoli L, et al Efficacy and feasibility of dose/scheduleadjusted Rd-R vs. continuous Rd in elderly and intermediate-fit newly diagnosed multiple myeloma (NDMM) patients: RV-MMPI-0752 phase III randomized study. Blood. 2018;132(Suppl 1). Abstract \#305 [ASH 2018 60th Meeting].

20. Durie BG, Hoering A, Sexton $R$, et al. Longer term follow up of the randomized phase III trial SWOG S0777: bortezomib, lenalidomide and dexamethasone vs. lenalidomide and dexamethasone in patients (pts) with previously untreated multiple myeloma without an intent for immediate autologous stem cell transplant (ASCT). Blood. 2018;132(Suppl 1). Abstract \#1992 [ASH 2018 60th Meeting].

21. Mateos M-V, Dimopoulos MA, Cavo M, et al. Daratumumab plus bortezomib, melphalan, and prednisone for untreated myeloma. N Engl J Med. 2018;378(6):518528.

22. Ocio EM, Otero PR, Bringhen S, et al Preliminary results from a phase I study of isatuximab (ISA) in combination with bortezomib, lenalidomide, dexamethasone (VRd) in patients with newly diagnosed multiple myeloma (NDMM) noneligible for transplant. Blood. 2018;132(Suppl 1). Abstract \#595 [ASH 2018 60th Meeting].

23. Facon T, Kumar S, Plesner T, et al Daratumumab plus lenalidomide and dexamethasone for untreated myeloma. N Engl J Med. 2019;380(22):2104-2115.

24. Dimopoulos MA, Mateos M-V, Cavo M, et al. One-year update of a phase 3 randomized study of daratumumab plus bortezomib, melphalan, and prednisone (DVMP) versus bortezomib, melphalan, and prednisone (VMP) in patients (pts) with transplant-ineligible newly diagnosed multiple myeloma (NDMM): Alcyone. Blood. 2018;132(Suppl 1). Abstract \#156 (ASH 2018 60th Meeting]. 